\title{
Successful medical treatment for a Crohn's disease patient with a perforation by a second-generation patency capsule
}

\section{다)(1) $\odot$}

\author{
Authors \\ Mikihiro Fujiya ${ }^{4}$, Toshikatsu Okumura ${ }^{4}$ \\ Institutions \\ 1 Department of Gastroenterology, International \\ University of Health and Welfare Hospital, \\ Nasushiobara, Japan \\ 2 Department of Surgery, International University of \\ Health and Welfare Hospital, Nasushiobara, Japan \\ 3 Konuma Gastrointestinal Clinic, Nasushiobara, Japan \\ 4 Division of Gastroenterology and Hematology/Oncology \\ Department of Medicine, Asahikawa Medical University, \\ Asahikawa, Japan
}

Hiroki Tanabe ${ }^{1}$, Katsuyoshi Ando ${ }^{1}$, Hironori Ohdaira², Yutaka Suzuki ${ }^{2}$, Ichiro Konuma ${ }^{3}$, Nobuhiro Ueno ${ }^{4}$,

submitted 18.4 .2018

accepted after revision 9.8.2018

\author{
Bibliography \\ DOI https://doi.org/10.1055/a-0752-9903 | \\ Endoscopy International Open 2018; 06: E1436-E1438 \\ (c) Georg Thieme Verlag KG Stuttgart · New York \\ ISSN 2364-3722
}

\author{
Corresponding author \\ Hiroki Tanabe, Division of Gastroenterology and \\ Hematology/Oncology, Department of Medicine, \\ Asahikawa Medical University, Midorigaoka- \\ Higashi 2-1-1-1, Asahikawa Hokkaido 078-8510, Japan \\ Fax: +81-166-68-2469 \\ tant@asahikawa-med.ac.jp
}

\section{ABSTRACT}

Background and study aims Symptomatic capsule retention is a very rare adverse event following patency capsule, and the vast majority of cases are resolved without either surgical or endoscopic intervention. We herein describe a rare case of small bowel perforation after swallowing a patency capsule in a 37-year-old man suspected of having Crohn's disease.

\section{Introduction}

Capsule endoscopy is useful for diagnosing small-bowel conditions in patients suspected of having Crohn's disease (CD). It is less invasive but may be associated with severe complications, such as capsule retention, which is usually caused by small bowel stricture [1]. A patency capsule (PC), which is a non-diagnostic capsule the same size as diagnostic capsule endoscopy, is used to determine the risk of capsule retention [2,3]. Successful excretion of the ingested PC after a predetermined time ( $40 \mathrm{~h}$ for the first-generation capsules and $30 \mathrm{~h}$ for the secondgeneration capsules) indicates that diagnostic capsule endoscopy can be safely performed [4,5].

The first-generation PC starts to dissolve spontaneously on contact with digestive secretions after 40 hours and completely shrinks between 80 hours to 100 hours. However, adverse events (AEs) with abdominal pain have been reported in up to $27.3 \%$ of patients according to the pertinent literature. A second-generation PC was therefore designed with two timer plugs, allowing for enhanced contact with the intestinal fluid to minimize the occurrence of AEs. Complications with the sec- ond-generation PCs have become rare, and severe AEs reportedly occur in from $0 \%$ to $3.7 \%$ of all cases [5].

We herein report a rare case of small bowel perforation after swallowing a PC in a patient suspected of having CD.

\section{Case report}

A 37-year-old man presented to our hospital for detailed examination and treatment of CD. He had complained of frequent diarrhea for more than 10 years and had undergone surgical treatment for hemorrhoids several years prior to this presentation. He complained of frequent hematochezia without abdominal pain or nausea and consulted a medical practitioner. A gastroenterologist performed colonoscopy, which showed longitudinal ulcers in the terminal ileum. As a result, the patient was suspected of having CD.

Computed tomography $(\mathrm{CT})$ revealed wall thickening of the terminal ileum without active enhancement by iodine contrast. A blood test showed a normal white blood cell (WBC) count $(8430 / \mu \mathrm{L})$ and $\mathrm{C}$-reactive protein level $(0.3 \mathrm{mg} / \mathrm{dL})$ with no anemia (hemoglobin $16.3 \mathrm{~g} / \mathrm{dL}$ ). Differential diagnosis included intestinal tuberculosis and drug-induced ileitis, except for CD. 


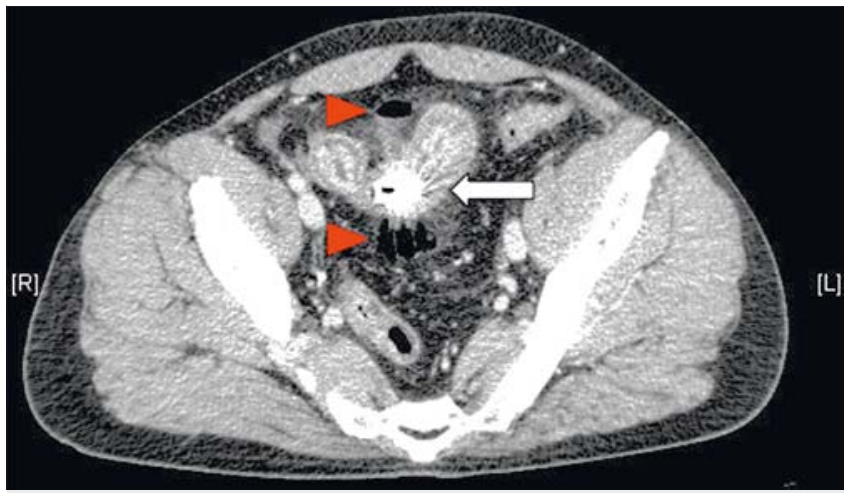

- Fig. 1 Computed tomography performed 9 hours after swallowing a patency capsule (PC) showing free air (red arrowheads) and the $\mathrm{PC}$ retained in the terminal ileum (arrow).

We then planned to perform capsule endoscopy to examine the small bowel, and a PC (Pillcam; Given Imaging, Yoqneam, Israel) examination was performed in advance to check for any stenosis that might cause retention of the capsule endoscopy. However, 4 hours after swallowing the PC, the patient developed abdominal pain and vomiting. When he arrived at our hospital with intolerable pain, a blood test showed an increased WBC count $(20,750 / \mu \mathrm{L})$ and $C T$ revealed localized free air near the PC retained at the terminal ileum with enhanced wall thickening ( $\triangleright$ Fig. 1). The patient was diagnosed with a minor perforation in the terminal ileum and hospitalized for bowel rest. Three days after receiving medical treatment with antibiotics (Melopenem $2 \mathrm{~g} \mathrm{q8hr),} \mathrm{excretion} \mathrm{of} \mathrm{the} \mathrm{PC} \mathrm{was} \mathrm{confirmed} \mathrm{by} \mathrm{plain}$ abdominal radiography ( $\mathbf{F i g . 2 a , ~} \mathbf{F i g . 2 b}$ ). The PC was spontaneously excreted within 72 hours, with no endoscopic or surgical intervention.

One month after the patient was discharged from our hospital, colonoscopy revealed mild stenosis ( $\mathbf{F i g . 3 a , ~} \mathbf{F i g . 3 b}$ ), and subsequent barium enteroclysis depicted longitudinal ulceration with a cobblestone appearance in the terminal ileum ( $\triangleright$ Fig.4a, $>$ Fig.4b). The patient was treated with anti-tumor necrosis factor-alpha antibody $(5 \mathrm{mg} / \mathrm{kg})$ without surgery.

\section{Discussion}

This was a very rare AE after swallowing a $P C$ in a patient suspected of having $C D$. The self-dissolving capsule was able to pass through the area of stenosis in the intestine. Short-term medical treatment without any intervention was effective for our case with a localized minor perforation.

A PC is usually safe for patients with $C D$ who have small intestinal stenosis, despite occasional AEs such as abdominal pain due to capsule retention and intestinal perforation $[3,6$, 7]. Several studies have reported the safety, reliability, and limitations of PCs, and only a few AEs have been reported with the second-generation PCs $[2,8]$. In previous reports, when retained PCs have caused severe symptoms, either endoscopic or surgical intervention was performed. [5]. In contrast, we were able to successfully treat the patient with $C D$ with bowel rest and appropriate antibiotics. In our case, because perfora-

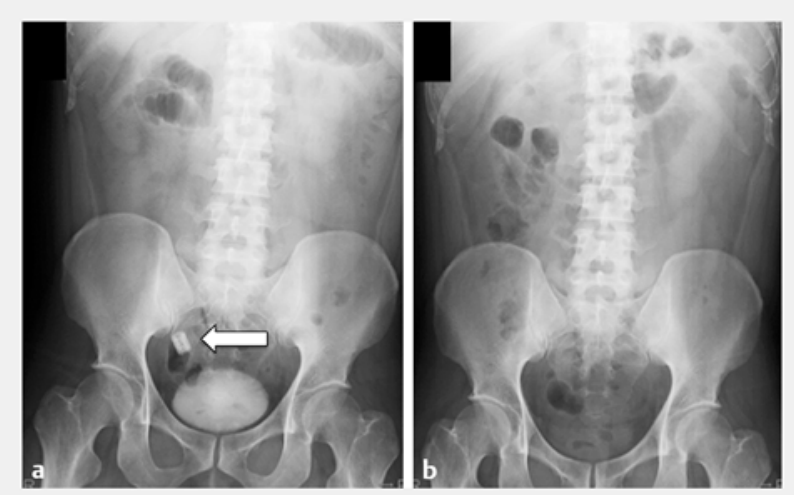

- Fig. 2a An abdominal X-ray obtained at 9 hours after swallowing a PC (arrow). b Three days after admission, the PC had been expelled.

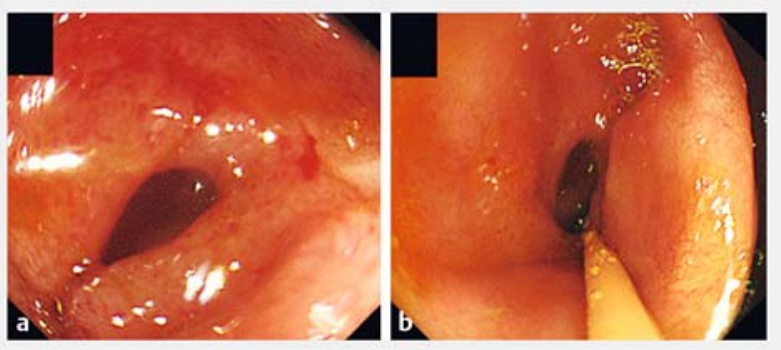

- Fig. 3a Colonoscopy showing stricture in the distal ileum and b taking a biopsy specimen.

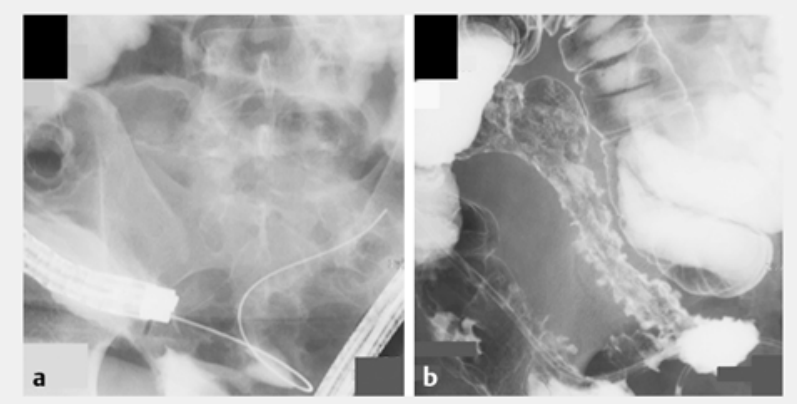

- Fig. 4a Colonoscopy at the end of the terminal ileum. A guidewire was inserted through the scope. b Retrograde enteroclysis showing longitudinal ulceration with a cobblestone appearance in the terminal ileum.

tion of the terminal ileum was minor, endoscopic intervention was not conducted to remove the retained $P C$ and the patient excreted it within 72 hours. Short-term careful medical treatment without intervention, therefore, may be a useful clinical strategy for cases in which localized intestinal perforation is caused by a PC. Because digestion of second-generation PCs begins at 30 hours and disintegrate within 100 hours, they are 
expected to be associated with fewer AEs and much milder symptoms in the short term than are first-generation PCs [4].

Guidelines for assessing inflammatory bowel disease recommend evaluation of small bowel patency before capsule endoscopy is performed, using a PC or cross-sectional imaging [1]. In our case, a PC was selected for a patient with suspected CD whose major complaint was hematochezia. No prestenotic dilatation was observed on CT.

Several other options for evaluating the severity of small intestinal CD have been proposed. Magnetic resonance enterography is a radiological imaging modality for assessing the small intestine after ingestion of an oral contrast agent [9]. It accurately depicts both intraluminal and extraluminal disease manifestations and predicts PC retention in patients with CD. Small bowel endoscopy is used to obtain biopsy specimens for pathological examination and for performing therapies such as balloon dilation for stenotic lesions of CD [10]. Direct observation of the mucosa allows for evaluation of disease activity and for a diagnosis to be made at an early stage. Application of these endoscopic techniques is limited in patients with active $C D$ or for those in the postoperative setting [1]. A novel imaging technique using a combination of endoscopic technique and CT may be useful for obtaining a detailed evaluation [11].

\section{Conclusion}

Capsule endoscopy is useful for evaluating small intestine CD by means of a minimally invasive modality, allowing us to visualize the entire intestine. PC is used to avoid complications such as retention. Our case report of a minor perforation in the small intestine using a second-generation PC suggests that caution is required regarding capsule retention and in selecting the optimal therapeutic options for medical treatment.
Competing interests

None

References

[1] Panes ], Bouhnik Y, Reinisch W et al. Imaging techniques for assessment of inflammatory bowel disease: joint ECCO and ESGAR evidence-based consensus guidelines. J Crohns Colitis 2013; 7: 556- 585

[2] Nemeth A, Kopylov U, Koulaouzidis A et al. Use of patency capsule in patients with established Crohn's disease. Endoscopy 2016; 48: $373-379$

[3] Gay G, Delvaux M, Laurent V et al. Temporary intestinal occlusion induced by a "patency capsule" in a patient with Crohn's disease. Endoscopy 2005; 37: 174-177

[4] Spada C, Spera G, Riccioni M et al. A novel diagnostic tool for detecting functional patency of the small bowel: the Given patency capsule. Endoscopy 2005; 37: 793-800

[5] Kopylov U, Nemeth A, Cebrian A et al. Symptomatic retention of the patency capsule: a multicenter real life case series. Endosc Int Open 2016; 4: E964 - 969

[6] Postgate AJ, Burling D, Gupta A et al. Safety, reliability and limitations of the given patency capsule in patients at risk of capsule retention: a 3-year technical review. Dig Dis Sci 2008; 53: 2732 - 2738

[7] Garg S, Anand R, Dubin E et al. Endoscopic management of retained patency capsules. Endoscopy 2014; 46: (Suppl. 01): E662-663

[8] Rasmussen B, Nathan T, Jensen MD. Symptomatic patency capsule retention in suspected Crohn's disease. J Crohns Colitis 2016; 10: $1445-1447$

[9] Rozendorn N, Klang E, Lahat A et al. Prediction of patency capsule retention in known Crohn's disease patients by using magnetic resonance imaging. Gastrointest Endosc 2016; 83: 182 - 187

[10] Yamagami H, Watanabe K, Kamata $\mathrm{N}$ et al. Small bowel endoscopy in inflammatory bowel disease. Clin Endosc 2013; 46: 321 - 326

[11] Tanabe H, Ito T, Inaba Y et al. Pilot study of endoscopic retrograde 3dimensional computed tomography enteroclysis for the assessment of Crohn's disease. Eur J Radiol Open 2017; 4: 58-62 